\title{
Performance comparison between the mycobacteria growth indicator tube system and löwenstein-Jensen medium in the routine detection of Mycobacterium tuberculosis at public health care facilities in Rio de Janeiro, Brazil: preliminary results of a pragmatic clinical trial*
}

\author{
Comparação do desempenho do sistema mycobacteria growth indicator \\ tube e meio Löwenstein-Jensen na detecção de rotina de Mycobacterium \\ tuberculosis em unidades do sistema único de saúde no Rio de Janeiro: \\ resultados preliminares de um ensaio clínico pragmático
}

\author{
Adriana da Silva Rezende Moreira, Gisele Huf, Maria Armanda Vieira, \\ Leila Fonseca, Monica Ricks, Afrânio Lineu Kritski
}

\begin{abstract}
In view of the fact that the World Health Organization has recommended the use of the mycobacteria growth indicator tube (MGIT) 960 system for the diagnosis of tuberculosis and that there is as yet no evidence regarding the clinical impact of its use in health care systems, we conducted a pragmatic clinical trial to evaluate the clinical performance and cost-effectiveness of the use of MGIT 960 at two health care facilities in the city of Rio de Janeiro, Brazil, where the incidence of tuberculosis is high. Here, we summarize the methodology and preliminary results of the trial.
\end{abstract}

(ISRCTN.org ldentifier: 1SRCTN79888843 [http://isrctn.org/])

Keywords: Controlled clinical trial; Tuberculosis; Diagnostic tests, routine.

\section{Resumo}

Em razão da recomendação da Organização Mundial da Saúde sobre o uso do sistema mycobacteria growth indicator tube (MGIT) 960 para o diagnóstico de tuberculose e da falta de evidências sobre o impacto clínico de sua incorporação em sistemas de saúde, um ensaio clínico pragmático está sendo conduzido para avaliar o desempenho clínico e a relação custo-efetividade do MGIT 960 em duas unidades do Sistema Único de Saúde na cidade do Rio de Janeiro, que tem uma elevada incidência de tuberculose. Apresentamos aqui, de forma sintética, o método e resultados preliminares do ensaio.

(ISRCTN.org ldentifier: 1SRCTN79888843 [http://isrctn.org/])

Descritores: Ensaio clínico controlado; Tuberculose; Testes diagnósticos de rotina.

Rapid diagnosis of tuberculosis is essential for reducing the length of time for which the chain of transmission is maintained and, therefore, the number of individuals infected by those with the disease. Sputum smear microscopy has low sensitivity, and the major problem with culture for mycobacteria is the long incubation period required. ${ }^{(1,2)}$ New tests, despite the high cost, can represent a breakthrough in the fight against the disease, especially in HIV-infected patients, or in patients treated at hospital level. ${ }^{(3)}$

In 2008, the World Health Organization ${ }^{(4)}$ recommended the use of solid media, such as the mycobacteria growth indicator tube (MGIT) system, because, in studies of diagnostic accuracy, the MGIT 960 system (Becton Dickinson, Sparks, MD, USA) has shown results similar to those observed with the use of the gold standard-

* Study carried out under the auspices of the Academic Tuberculosis Program, Federal University of Rio de Janeiro School of Medicine Hospital Universitário Clementino Fraga Filho, Instituto de Doenças do Tórax - HUCFF-IDT, Clementino Fraga Filho University Hospital/Thoracic Diseases Institute - Rio de Janeiro, Brazil.Correspondence to: Afrânio L. Kritski. Hospital Universitário Clementino Fraga Filho, Universidade Federal do Rio de Janeiro, Rua Rodolpho Paulo Rocco, 255, llha do Fundão, CEP 21541912, Rio de Janeiro, RJ, Brasil.

Tel. 5521 2562-2426. E-mail: kritskia@gmail.com

Financial support: This study received financial support via the Project CNPq/CT-Saúde/MS/SCTIE/DECIT, Grant no. 559081/2009. Submitted: 22 January 2013. Accepted, after review: 30 January 2013. 
Löwenstein-Jensen (LJ) solid medium,-whether in the detection of Mycobacterium tuberculosis or in the identification of strains resistant to the two most important drugs in the first-line antituberculosis treatment regimens-rifampin and isoniazid. In addition, the time to a positive result for MGIT 960 is shorter (10-15 days) than that for the test performed on $\mathrm{LJ}$, which ranges from 20 to 40 days.

Typically, when diagnostic kits are validated by the US Food and Drug Administration or by European regulatory agencies, the regulatory agencies in developing countries tend to approve those kits for marketing, provided that the same items contained in the products marketed in the country of origin are included in the usage instrument (directions for use). However, in recent years in Brazil, researchers affiliated with the Brazilian Tuberculosis Research Network have proposed that, before new diagnostic methods related to diseases of public health impact are granted approval, are available in the national market, and, especially, are used in the Sistema Único de Saúde (SUS, Brazilian Unified Health Care System), they be evaluated in terms of their clinical performance and cost-effectiveness, under routine conditions, through practical clinical trials. Through such studies, it is possible to evaluate interventions under conditions that are as close as possible to those under which such interventions are applied in clinical practice, supporting decision making, as opposed to so-called explanatory clinical trials, which are conducted under more controlled conditions and seek to answer questions about whether and how an intervention works, a requirement for products to be approved and marketed. Because practical studies are developed under routine conditions, they do not complicate the usual practice and do not compete with routine activities, thereby being inexpensive and securing voluntary collaboration from the team. ${ }^{(5)}$

The Brazilian Tuberculosis Research Network, with support from the Brazilian National Ministry of Health, have conducted a practical clinical trial to evaluate the clinical performance and cost-effectiveness of the use of MGIT 960 at two health care facilities in the city of Rio de Janeiro, Brazil, where the incidence of tuberculosis is high (85/100.000 population). The present study was motivated by the following considerations: the World Health Organization has recommended the use of MGIT 960 for the diagnosis of tuberculosis; to our knowledge, the literature provides no data on the evaluation of the clinical impact of the use of this diagnostic method or on the economic analysis of its use in health care systems ${ }^{(6)}$; this diagnostic method has not yet been used in the SUS in Brazil. The present study involved inpatients at the Universidade Federal do Rio de Janeiro (UFRJ, Federal University of Rio de Janeiro) Hospital Universitário Clementino Fraga Filho (HUCFF, Clementino Fraga Filho University Hospital) and outpatients at a secondary care facility-the Rio de Janeiro Municipal Department of Health Policlínica de Guadalupe (PG, Guadalupe Polyclinic). A cost-effectiveness analysis is currently under way. Below, we summarize the methodology and preliminary results of the clinical trial. The research project was approved by the UFRJ Research Ethics Committee (Protocol no. 020/07), and the clinical trial is registered with the identifier 1SRCTN79888843.

At the two study sites, the main inclusion criterion was suspicion of tuberculosis in over-16year-olds; those who were already being treated for tuberculosis were excluded. The MGIT 960 system was compared with the traditional method (LJ). The primary outcome was a change in the initial approach (initiation or discontinuation of treatment) within two months after inclusion of the patient in the study. The participants were randomized, and there was no blinding, with the exception of the outcome assessors. Measures were taken to ensure concealment of the randomization.

At the HUCFF and at the PG, respectively, 427 and 266 patients with suspected tuberculosis were randomized between April of 2008 and October of 2010 and between April of 2008 and July of 2009. At both sites, the randomization process was successful in producing groups with similar sociodemographic and clinical characteristics. At the HUCFF, the study population consisted of inpatients, whereas, at the PG, it consisted of outpatients. The mean age of the participants was slightly higher at the HUCFF than at the PG (51 vs. 45), as was the proportion of male participants (55\% vs. 50\%). In addition, presumptive identification of tuberculosis, performed by an experienced, well-trained nurse at inclusion in the study, was much greater at the HUCFF than at the PG (20\% vs. $4 \%)$. The preliminary results are shown in Table 1. 
Table 1 - Performance comparison between the mycobacteria growth indicator tube 960 system and Löwenstein-Jensen solid medium at the Clementino Fraga Filho University Hospital and at the Guadalupe Polyclinic, Rio de Janeiro, Brazil. ${ }^{a}$

\begin{tabular}{|c|c|c|c|c|}
\hline \multirow[t]{3}{*}{ Variable } & \multicolumn{2}{|c|}{ HUCFF } & \multicolumn{2}{|c|}{ PG } \\
\hline & MGIT & $\mathrm{LJ}$ & MGIT & $\mathrm{LJ}$ \\
\hline & $(n=214)$ & $(n=213)$ & $(n=134)$ & $(n=132)$ \\
\hline Positive smear result & $6(2.8)$ & $10(4.7)$ & 29 (21.6) & $24(19.0)$ \\
\hline $\begin{array}{l}\text { Positive culture for } \\
\text { M. tuberculosis }\end{array}$ & $25(11.7)$ & $20(10.0)$ & $38(28.3)$ & $31(23.5)$ \\
\hline Time to result, days ${ }^{b}$ & $10.7 \pm 5.7(4-25)$ & $31.7 \pm 8.9(19-49)$ & $10.6 \pm 7.0(2-31)$ & $29.5 \pm 8.9(14-55)$ \\
\hline
\end{tabular}

Some relevant aspects were observed. Sputum smear microscopy was ineffective for the inpatients at the HUCFF, who were, in general, paucibacillary. The results seem to indicate an advantage for the MGIT in the two study sites, given that the reporting of the results to the attending physician occurred sooner. At present, the final analysis of the clinical performance of the tests in terms of patient-centered outcomes (morbidity, mortality, length of hospital stay, unnecessary medication use, and, in patients diagnosed with tuberculosis, bacteriological conversion and pharmacological treatment response) is under way.

\section{References}

1. World Health Organization. Global Tuberculosis Control: Surveillance, Planning, Financing. Geneva: World Health Organization; 2010.
2. Fair E, Hopewell PC, Pai M. International Standards for Tuberculosis Care: revisiting the cornerstones of tuberculosis care and control. Expert Rev Anti Infect Ther. 2007;5(1):61-5. PMid:17266454. http://dx.doi. org/10.1586/14787210.5.1.61

3. World Health Organization. New Laboratory Diagnostic Tools for Tuberculosis control. Geneva: World Health Organization; 2008.

4. World Health Organization. Use of liquid TB culture and drug susceptibility testing (DST) in low and medium income settings. Geneva: World Health Organization; 2007.

5. Huf G, Kritski A. Evaluation of the clinical utility of new diagnostic tests for tuberculosis: the role of pragmatic clinical trials. J Bras Pneumol. 2012;38(2):23745. PMid:22576433. http://dx.doi.org/10.1590/ S1806-37132012000200014

6. Ferrante di Ruffano L, Hyde CJ, McCaffery KJ, Bossuyt PM, Deeks JJ. Assessing the value of diagnostic tests: a framework for designing and evaluating trials. BMJ. 2012;344:e686. PMid:22354600. http://dx.doi.org/10.1136/ bmj.e686

\section{About of the authors}

\section{Adriana da Silva Rezende Moreira}

Nurse. Federal University of Rio de Janeiro Hospital Universitário Clementino Fraga Filho, Instituto de Doenças do Tórax HUCFF-IDT, Clementino Fraga Filho University Hospital/Thoracic Diseases Institute - Rio de Janeiro, Brazil.

\section{Gisele Huf}

Researcher. Oswaldo Cruz Foundation National Institute of Quality Control in Health, Rio de Janeiro, Brazil.

\section{Maria Armanda Vieira}

Researcher. Academic Tuberculosis Program, Federal University of Rio de Janeiro Hospital Universitário Clementino Fraga Filho, Instituto de Doenças do Tórax - HUCFF-IDT, Clementino Fraga Filho University Hospital/Thoracic Diseases Institute - Rio de Janeiro, Brazil.

\section{Leila Fonseca}

Researcher. Academic Tuberculosis Program, Federal University of Rio de Janeiro Hospital Universitário Clementino Fraga Filho, Instituto de Doenças do Tórax - HUCFF-IDT, Clementino Fraga Filho University Hospital/Thoracic Diseases Institute - Rio de Janeiro, Brazil.

\section{Monica Ricks}

Researcher. Academic Tuberculosis Program, Federal University of Rio de Janeiro Hospital Universitário Clementino Fraga Filho, Instituto de Doenças do Tórax - HUCFF-1DT, Clementino Fraga Filho University Hospital/Thoracic Diseases Institute - Rio de Janeiro, Brazil.

\section{Afrânio Lineu Kritski}

Full Professor of Tuberculosis and Pulmonology. Federal University of Rio de Janeiro School of Medicine, Rio de Janeiro, Brazil. 